\title{
RETRACTED ARTICLE: Ten-year survival rates of methylprednisolone plus cyclophosphamide followed by mycophenolate mophetyl of azathiopurine for progressive systemic sclerosis patients
}

\author{
Metin Isik • Ali Akdoğan • Abdullah Agit • \\ İsmail Doğan · Levent Kılınç • Meral Çalgüneri
}

Received: 3 August 2011/Accepted: 22 October 2011/Published online: 19 November 2011

(C) Springer-Verlag 2011

This article has been retracted at the request of the authors.

The authors made a serious statistical error which unfortunately invalidates their results.

M. Isik ( $)$ · A. Akdoğan · İ. Doğan · L. Kılınç · M. Çalgüneri Division of Rheumatology, Department of Internal Medicine, Hacettepe University Hospital, 06100 Sihhiye Ankara, Turkey e-mail: metin1721978@yahoo.com

\section{A. Agit}

Department of Internal Medicine, Hacettepe University, Ankara, Turkey 